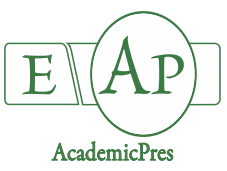

Artem A et al. (2021)

Notulae Botanicae Horti Agrobotanici Cluj-Napoca

Volume 49, Issue 2, Article number 12345

DOI:10.15835/nbha49212345

Research Article

\title{
Effect of grape yield and maceration time on phenolic composition of 'Fetească neagră' organic wine
}

\author{
Victoria ARTEM ${ }^{1}$, Arina O. ANTOCE ${ }^{2 *}$, Elisabeta I. GEANA², \\ Aurora RANCA ${ }^{1}$ \\ ${ }^{1}$ Research Station for Viticulture and Oenology Murfatlar, 02 Calea Bucuresti Street, 905100, Murfatlar, Constanta, \\ Romania; artemvictoria@yahoo.com \\ ${ }^{2}$ University of Agronomic Sciences and Veterinary Medicine of Bucharest, Faculty of Horticulture, Department of Bioengineering of \\ Horti-Viticultural Systems, 59 Marasti Ave.,Sector 1,011464, Bucharest,Romania; arina.antoce@horticultura-bucuresti.ro; \\ aantoce@yahoo.com (*corresponding author) \\ ${ }^{3}$ National R\&D Institute for Cryogenics and Isotopic Technologies-ICIT Rm. Valcea, 4th Uzinei STREET, po Raureni, Box 7 , \\ 240050 RM. Valcea, Romania; irina.geana@icsi.ro
}

\begin{abstract}
The phenolic composition of wine is mostly determined by the accumulation of the phenolic compounds in the grapes, as well as their extraction into wine. To increase their concentration in grapes, yield reduction is usually performed by pruning, while to increase the extraction in wines, the maceration on skins is extended for longer periods of time. The present study focuses on the possibilities to apply both strategies to improve the polyphenol composition of organic red wines of Romanian variety 'Fetească neagră', which stands to benefit more from technological interventions than other varieties, which naturally accumulate higher phenol concentrations in the grapes. In the vineyard three experimental pruning variants were made, with 20 , 28 and 36 buds/vine, while for wine, maceration was performed for either 8 or 16 days for each grape variant. The phenolic profiles of wines were determined by HPLC methods. The main anthocyanidins, such as malvidin, petunidin, delphinidin, peonidin and cyanidin, as well as the acylated and coumaroylated derivatives of malvidin and peonidin were quantitatively determined. Some other phenolic compounds, of various classes, such as gallic, p-benzoic, p-coumaric and ferulic acid, catechin, epicatechin, myricetin, quercetin and transresveratrol were also determined. The quality of the organic 'Fetească neagră' wines depended highly on the vintage, but yield reduction and the extension of skin maceration duration were especially beneficial in the less favourable year, when classical technologies lead to less accumulation of sugars, colour and other polyphenols. Concomitant application of both strategies led to the best results, irrespective of the year.
\end{abstract}

Keywords: cultivar; green pruning; maceration-fermentation; organic grapes

\section{Introduction}

At present more and more consumers are choosing their foods based on their nutritional value, safety and even environmental impact. For this reason, the segment of population buying organic food products is at an all-time high, including in Romania, where $80 \%$ of the tested consumers believe that the organic food is

Received: 15 Apr 2021. Received in revised form: O8 Jun 2021. Accepted: 16 Jun 2021. Published online: 18 Jun 2021.

From Volume 49, Issue 1, 2021, Notulae Botanicae Horti Agrobotanici Cluj-Napoca journal uses article numbers in place of the traditional method of continuous pagination through the volume. The journal will continue to appear quarterly, as before, with four annual numbers. 
healthier, while also $75 \%$ of them understand that organic agriculture contributes to environmental protection (Petrescu et al., 2015). This trend is also seen in viticulture products. At present, organic viticulture surfaces have increased worldwide up to 3 times higher than 10 years ago, while organic winemaking enjoys the largest recognition so far (Willer and Lernoud, 2017). Organic wines are more accessible and of a higher proven quality (Antoce, 2019), especially since 2012 when the European legislation on organic wines entered in force (Reg. EU 203,2012). Organic production methods are rooted in the nature's principles of production, which means working in harmony with nature rather than trying to conquer it (Ozabagaoglu et al., 2007), thus conferring sustainability.

Although the new generations are more interested in organic products for their link to sustainability, they are also looking for products perceived as healthier. Consumers are more easily accepting organic wines when these are also containing more components linked to a better human health. As it is very well known, aside from alcohol, wine contains several polyphenolic compounds which have been shown to have multiple positive effects on health (Hussain et al., 2016; Antoce and Stockley, 2019). Polyphenols from grapes and wines have proven beneficial effects for cardiovascular disease, diabetes, liver function, cognitive function, on microbiota and other microbial-related diseases and even for some forms of cancer (Antoce and Stockley, 2019). Among other European countries which are investing in organic viticulture, Romania has started to be more active too. Data regarding Romanian organically produced wines are scarce, but there are some researchers focusing on the determination of the health-related parameters and characteristics of our wines (Busuricu et al., 2013; Colibaba et al., 2019). As already stated, to meet the demand for wines more beneficial for human health, one of the main preoccupations is to increase the concentration of polyphenolic compounds. This is especially valid for the case of grapes with naturally lower accumulation of phenolic compounds, but all the wines/varieties can benefit from an increase. This goal can be achieved by interventions in the vineyard, as well as in the wine technology. By adjusting the canopy size and numbers of shoots and clusters per vine through the level of the pruning applied, it is possible to significantly influence the grape yield, overall fruit quality and its composition (Rahmani et al., 2015). Winemaking techniques can also play an important role in the final polyphenol composition, the extraction from the grape skins and seeds being highly influenced by the time of contact during the maceration and fermentation processes, as well as other technological steps such as pressing, maturation in contact with oak, fining and bottle aging (Ignat et al., 2017; Luchian et al., 2018). Macerationfermentation is the most critical stage in the red winemaking process. It is essential for obtaining high quality red wines, but is also the one that requires the most energy and workforce. It is estimated that about $64.3 \%$ of the total energy needed to produce wine is consumed during the maceration-fermentation stage (Genc et al., 2017).

The goal of this study was to optimize the phenolic composition and overall quality of red wines produced from organically grown 'Fetească neagră' grapes, in the vineyard by regulating the yield through pruning and in the winery by prolonging the usual period of maceration-fermentation on skins from 8 to 16 days.

\section{Materials and Methods}

\section{Biological material}

The grape used for the wine production is 'Fetească neagră' (Vitis vinifera cv.), a Romanian autochthonous variety, which has gained more and more recognition for its quality wines (Antoce and Cojocaru, 2018). In Murfatlar region, this variety accumulates sufficient polyphenolic compounds, around $1600 \mathrm{mg} \mathrm{L}^{-1} \mathrm{GAE}$, to give the wines a strong and stable colour, but does not reach the level naturally accumulated by 'Cabernet Sauvignon', which is around 2,100 $\mathrm{mg} \mathrm{L}^{-1}$ GAE (Dobrinas et al., 2012). At the Research and Development Station Murfatlar, the 'Fetească neagră' cultivar has been grown and certified in accordance to the organic production principles and regulations (Reg. EU 848, 2018) since 2009 on a surface 
of 8.1 ha. The experimental plots are placed on a parcel with N-S exposition, slope of 3-5\%, soil of chalky chernozem type with clay texture. The vine is planted on the rootstock Vitis berlandieri $\times V$. riparia Oppenheim 4 selection, clone $\mathrm{SO}_{4^{-4}}$ and trained with semi-high stems of $70 \mathrm{~cm}$ and bilateral cordons with cane pruning. The density of the plantation is 4,132 vines/ha, with $2.2 \mathrm{~m}$ between rows and $1.1 \mathrm{~m}$ between trunks. Each experimental plot consisted of 3 rows of vines for each variant and repetition. There were three experimental variants: control variant (C) pruned with a bud-load of 28 buds/vine, variant T1 with 20 buds/vine and variant $\mathrm{T} 2$ with 36 buds/vine. For each variant of 21 vines, three repetitions were made, arranged by randomized block design. The pruning was performed in March, when the danger of freezing and late frost had mostly passed and the mounded soil used for winter vine protection was removed.

Vintage

The grapes were manually harvested at a sugar concentration of 23.2 Brix in 2016 , September $12^{\text {th }}$ and 20.5 Brix in 2017, September $13^{\text {th }}$. The year of 2017 was less favourable, with an average of sunshine during the growing season of 1,527 hours and rainfall of $333 \mathrm{~mm}$ as compared to 2016 with sunshine of 1,715 hours and rainfall of $284 \mathrm{~mm}$.

\section{Wine production}

For each variant a total quantity of approximately $200 \mathrm{~kg}$ of grapes has been picked, transported to the pilot winemaking station of the research unit and vinified by a classical maceration-fermentation technology. The destemming and crushing, with an electrically-powered laboratory equipment (from Grifo, Italy) of processing volume $1500 \mathrm{~kg} \mathrm{~h}^{-1}$, were followed by the maceration of the mash in recipients of $120 \mathrm{~L}$ volume. For an optimum skin contact, the must and solid parts were homogenized 3-4 times a day, by manually punching down the cap formed from the grape skins and mixing it with the liquid. Maceration-fermentation temperature was kept under $25^{\circ} \mathrm{C}$ for the entire process. The solid parts separation was performed by a hydraulic wine press (from Grifo, Italy), with maximum pressure of 3 bar, at 8 days and 16 days of contact, respectively. The separated must was then introduced in $25 \mathrm{~L}$ demijohns and allowed to continue fermentation to dryness with the native yeasts and to start malolactic fermentation as well. After malolactic fermentation was completed the wine was racked from the lees, treated with a low dose of sulphur dioxide $50 \mathrm{mg} \mathrm{L}^{-1}$ and bottled in $0.75 \mathrm{~L}$ glass recipients. All the steps of the winemaking were performed in compliance with the rules for organic wines included in the European Regulation 203/2012, which are designed to respect the natural traits of the product, while keeping the wine diversity and quality.

\section{Standard chemical analyses}

To assess the quality of the wines and compliance with the regulations, the main physicochemical parameters have been determined in accordance to the methods provided by OIV in the Compendium of International Methods of Analysis (2008) and by ASRO (2012) in their standards. The tested parameters included the alcoholic strength (\% vol.), reducing sugar $\left(\mathrm{g} \mathrm{L}^{-1}\right)$, total acidity $\left(\mathrm{g} \mathrm{L}^{-1}\right.$ tartaric acid), volatile acidity ( $\mathrm{g} \mathrm{L}^{-1}$ acetic acid), total dry extract $\left(\mathrm{g} \mathrm{L}^{-1}\right)$, non-reducing dry extract $\left(\mathrm{g} \mathrm{L}^{-1}\right)$, total $\mathrm{SO}_{2}\left(\mathrm{mg} \mathrm{L}^{-1}\right)$ and free $\mathrm{SO}_{2}(\mathrm{mg}$ $\left.\mathrm{L}^{-1}\right)$.

\section{HPLC analysis of phenolic compounds}

To determine in more detail the wine quality and its potential to have a beneficial effect on the health, specific phenolic compounds were also analysed.

Determination of these individual polyphenols was performed using an UltiMate 3000 HPLC from Thermo Finnigan, equipped with an autosampler, a LC Pump (Quaternary gradient) and a Chromeleon Workstation. Detection was made at $280 \mathrm{~nm}$ using a Diode Array Detector (DAD). Phenolic acids (gallic, pbenzoic, p-coumaric and ferulic acid), flavanols (catechin and epicatechin), flavonols (myricetin, quercetin) and trans-resveratrol were determined using a previously published HPLC method (Geana et al., 2016). Also, 
the most important five anthocyanidin mono-glucosides (malvidin, petunidin, delphinidin, peonidin and cyanidin), along with malvidin and peonidin acylated and coumaroylated derivatives were assessed by OIV recommended HPLC methods (2008). Malvidin-3-O-glucoside (oenin) was identified using a calibration curve obtained with a standard reagent (Sigma Aldrich), while the other anthocyanins were identified, as presented in the OIV method, based on their order of elution from the C18 column and expressed as $\mathrm{mg} \mathrm{L}^{-1}$ malvidin-3-O-glucoside.

\section{Statistical analysis}

To determine if significant influences are incurred by the vintage, grape yield or the length of the maceration period, analysis of variance (One-way ANOVA) has been performed and the mean differences of the various samples were compared by a post-hoc test (Duncan multiple mean comparison test). The confidence level used in the statistical analysis was $95 \%$, which means that the maximum admissible probability of a type I error was 5\%. PCA analysis was performed with Origin 2018 software from OriginLab Corporation, USA.

\section{Results and Discussion}

The chemical analyses of the red wine samples produced in both years of harvest are presented in Tables 1 (2016) and Table 2 (2017). The parameters are reported for the three bud-load variants (C, T1 and T2) and, for each bud-load variant, for the wines macerated for 8 days (V1) and 16 days (V2).

The results show that the wines recorded a normal to high alcohol concentration in both years, ranging, in accordance to the experimental variants, from 11.2 to $12.7 \%$ vol. in 2016 and between 12.9 and $13.4 \%$ vol. in 2017. The variants with reduced bud-load (T2=20 bud/vine) recorded significantly higher values for the alcohol in wine compared to the control and to the variant with higher yield, due to a better sugar accumulation in the berries. In 2016 the conditions were more favourable for the sugar accumulation, thus the variant T2 did not differ significantly from the control $(\mathrm{T} 1>\mathrm{C}=\mathrm{T} 2)$, but in 2017, all the variants were different in this respect ( $11>\mathrm{C}>\mathrm{T} 2)$, showing that in less favourable years a high yield has a negative effect on the final quality. In both years the increase in alcohol concentration in the variant with the lowest grape yield (T1) was by about $4.3 \%$ as compared to the control (C). A tendency for a slight increase in the alcohol concentration is observed also for the wines macerated-fermented for 16 days as compared to 8 days, but the mean values differed significantly only in the case of control.

The sugar which remained in wine after the fermentation did not differ significantly among variants (2.2-2.6 $\left.\mathrm{g} \mathrm{L}^{-1}\right)$, as they were all fermented to dryness.

The fact that the high alcohol concentration is correlated with quality is demonstrated here by the fact that a similar tendency is observed for the non-reducing dry extract, which is higher in the samples produced from the low-yield variants. In the high yield variants, the average extract decreases by approximately $1 \mathrm{~g} \mathrm{~L}^{-1}$ as compared to control and $2 \mathrm{~g} \mathrm{~L}^{-1}$ as compared to the low-yield variants. This fact is not negligible, as a difference of $1 \mathrm{~g} \mathrm{~L}^{-1}$ dry extract may be perceivable by sensory analysis and, coupled with a lower alcoholic concentration, gives the impression of a lighter-bodied wine. Generally, the naturally acquired extract of red wines produced in Romanian vineyards have values between $18-30 \mathrm{~g} \mathrm{~L}^{-1}$, depending on the specificity of the vineyard, the variety, the conditions of the year, but also depending on the technology applied (Antoce, 2007).

The total acidity also shows that 2017 was a less favourable year for grape maturation, with values of 6.25-6.83 g L-1 $\mathrm{C}_{4} \mathrm{H}_{6} \mathrm{O}_{6}$, as compared to $4.81-5.56 \mathrm{~g} \mathrm{~L}^{-1}$ in 2016. The yield, however, did not induce significant differences among variants. 
Table 1. Chemical composition of wines obtained in experimental variants of 'Fetească neagră', vintage 2016

\begin{tabular}{|c|c|c|c|c|c|c|}
\hline \multirow[b]{2}{*}{ Compound } & \multicolumn{2}{|c|}{$\mathrm{C}=28 \mathrm{buds}$} & \multicolumn{2}{|c|}{$\mathrm{T} 1=20 \mathrm{buds}$} & \multicolumn{2}{|c|}{$\mathrm{T} 2=36$ buds } \\
\hline & $\begin{array}{l}\mathrm{V} 1=8 \text { days } \\
\text { maceration }\end{array}$ & $\begin{array}{l}\mathrm{V} 2=16 \text { days } \\
\text { maceration }\end{array}$ & $\begin{array}{l}\mathrm{V} 1=8 \text { days } \\
\text { maceration }\end{array}$ & $\begin{array}{l}\mathrm{V} 2=16 \text { days } \\
\text { maceration }\end{array}$ & $\begin{array}{l}\mathrm{V}=8 \text { days } \\
\text { maceration }\end{array}$ & $\begin{array}{l}\mathrm{V} 2=16 \text { days } \\
\text { maceration }\end{array}$ \\
\hline \multicolumn{7}{|c|}{ General composition } \\
\hline Alcoholic strength (\% vol.) & $13.0 \pm 0.1 \mathrm{c}$ & $13.27 \pm 0.1 \mathrm{ab}$ & $13.36 \pm 0.1 \mathrm{a}$ & $13.41 \pm 0.1 \mathrm{a}$ & $12.9 \pm 0.2 \mathrm{c}$ & $13.1 \pm 0.2 \mathrm{bc}$ \\
\hline Reducing sugar $\left(\mathrm{g} \mathrm{L}^{-1}\right)$ & $2.4 \pm 0.1 \mathrm{bc}$ & $2.3 \pm 0.1 \mathrm{c}$ & $2.6 \pm 0.2 \mathrm{a}$ & $2.5 \pm 0.1 \mathrm{ab}$ & $2.5 \pm 0.1 \mathrm{ab}$ & $2.3 \pm 0.1 \mathrm{c}$ \\
\hline Total acidity ( $\mathrm{g} \mathrm{L}^{-1}$ tartaric acid) & $5.11 \pm 0.22 \mathrm{ab}$ & $4.81 \pm 0.26 \mathrm{~b}$ & $5.04 \pm 0.33 b$ & $5.19 \pm 0.25 \mathrm{ab}$ & $5.04 \pm 0.31 \mathrm{~b}$ & $5.56 \pm 0.19 \mathrm{a}$ \\
\hline Volatile acidity (g L ${ }^{-1}$ acetic acid) & $0.46 \pm 0.11 \mathrm{a}$ & $0.46 \pm 0.10 \mathrm{a}$ & $0.49 \pm 0.10 \mathrm{a}$ & $0.49 \pm 0.12 \mathrm{a}$ & $0.41 \pm 0.15 \mathrm{a}$ & $0.48 \pm 0.18 \mathrm{a}$ \\
\hline Total dry extract $\left(\mathrm{g} \mathrm{L}^{-1}\right)$ & $27.1 \pm 0.2 \mathrm{c}$ & $27.6 \pm 0.1 \mathrm{bc}$ & $28.0 \pm 0.2 \mathrm{ab}$ & $28.6 \pm 0.2 \mathrm{a}$ & $26.1 \pm 0.1 \mathrm{~d}$ & $26.8 \pm 0.2 \mathrm{~cd}$ \\
\hline Non-reducing dry extract $\left(\mathrm{g} \mathrm{L}^{-1}\right)$ & $24.7 \pm 0.2 \mathrm{c}$ & $25.3 \pm 0.2 \mathrm{~b}$ & $25.4 \pm 0.1 \mathrm{~b}$ & $26.1 \pm 0.1 \mathrm{a}$ & $23.8 \pm 0.1 \mathrm{c}$ & $24.3 \pm 0.2 \mathrm{~d}$ \\
\hline Total $\mathrm{SO}_{2}\left(\mathrm{mg} \mathrm{L}^{-1}\right)$ & $78.2 \pm 3.1 \mathrm{a}$ & $79.5 \pm 3.8 \mathrm{a}$ & $77.2 \pm 3.6 \mathrm{a}$ & $80.0 \pm 4.2 \mathrm{a}$ & $76.8 \pm 3.7 \mathrm{a}$ & $81.2 \pm 3.5 \mathrm{a}$ \\
\hline Free $\mathrm{SO}_{2}\left(\mathrm{mg} \mathrm{L}^{-1}\right)$ & $26.5 \pm 1.8 \mathrm{ab}$ & $25.3 \pm 1.6 \mathrm{~b}$ & $28.1 \pm 2.0 \mathrm{ab}$ & $29.5 \pm 2.5 \mathrm{ab}$ & $28.6 \pm 2.8 \mathrm{ab}$ & $30.1 \pm 2.4 \mathrm{a}$ \\
\hline \multicolumn{7}{|c|}{ Phenolic compounds } \\
\hline \multicolumn{7}{|c|}{ Phenolic acids $\left(\mathrm{mg} \mathrm{L}^{-1}\right)$} \\
\hline Gallic acid & $9.77 \pm 1.9 \mathrm{de}$ & $27.88 \pm 2.1 \mathrm{~b}$ & $13.42 \pm 2.1 \mathrm{~d}$ & $34.87 \pm 2.7 \mathrm{a}$ & $7.76 \pm 1.2 \mathrm{c}$ & $22.16 \pm 2.1 \mathrm{c}$ \\
\hline p-Hydroxybenzoic acid & $5.5 \pm 1.0 \mathrm{~b}$ & $7.04 \pm 1.5 \mathrm{ab}$ & $6.29 \pm 1.8 \mathrm{ab}$ & $8.34 \pm 1.6 \mathrm{a}$ & $2.05 \pm 0.9 \mathrm{c}$ & $6.99 \pm 1.6 \mathrm{ab}$ \\
\hline p-Coumaric acid & $1.44 \pm 0.8 \mathrm{a}$ & $1.16 \pm 0.8 \mathrm{a}$ & $1.78 \pm 0.9 \mathrm{a}$ & $1.23 \pm 0.7 \mathrm{a}$ & $1.0 \pm 0.2 \mathrm{a}$ & $0.84 \pm 0.2 \mathrm{a}$ \\
\hline Ferulic acid & $4.37 \pm 1.3 \mathrm{bc}$ & $6.31 \pm 1.6 \mathrm{ab}$ & $6.03 \pm 1.3 \mathrm{ab}$ & $7.52 \pm 1.5 \mathrm{a}$ & $2.83 \pm 1.1 \mathrm{c}$ & $4.13 \pm 1.2 \mathrm{bc}$ \\
\hline \multicolumn{7}{|l|}{ Flavanols $\left(\mathrm{mg} \mathrm{L}^{-1}\right)$} \\
\hline Catechin & $7.01 \pm 1.7 \mathrm{bc}$ & $13.69 \pm 2.0 \mathrm{a}$ & $9.07 \pm 2.1 \mathrm{~b}$ & $14.61 \pm 2.3 \mathrm{a}$ & $5.52 \pm 1.8 \mathrm{c}$ & $10.04 \pm 2.2 \mathrm{~b}$ \\
\hline Epicatechin & $1.99 \pm 0.8 \mathrm{ab}$ & $2.17 \pm 0.9 \mathrm{ab}$ & $2.71 \pm 1.5 \mathrm{ab}$ & $3.03 \pm 1.0 \mathrm{a}$ & $0.87 \pm 0.1 \mathrm{~b}$ & $2.09 \pm 1.0 \mathrm{ab}$ \\
\hline \multicolumn{7}{|l|}{ Flavonols $\left(\mathrm{mg} \mathrm{L}^{-1}\right)$} \\
\hline Myricetin & $4.57 \pm 1.6 \mathrm{a}$ & $3.94 \pm 1.5 \mathrm{a}$ & $5.11 \pm 1.4 \mathrm{a}$ & $4.69 \pm 1.2 \mathrm{a}$ & $4.16 \pm 1.8 \mathrm{a}$ & $3.61 \pm 1.2 \mathrm{a}$ \\
\hline Quercetin & $3.52 \pm 1.2 \mathrm{~b}$ & $1.21 \pm 0.4 \mathrm{c}$ & $5.94 \pm 1.7 \mathrm{a}$ & $2.9 \pm 0.9 \mathrm{bc}$ & $3.44 \pm 1.1 \mathrm{~b}$ & $1.8 \pm 0.2 \mathrm{bc}$ \\
\hline \multicolumn{7}{|l|}{ Stilbens $\left(\mathrm{mg} \mathrm{L}^{-1}\right)$} \\
\hline Trans-resveratrol & $1.18 \pm 0.5 \mathrm{a}$ & $1.48 \pm 0.8 \mathrm{a}$ & $1.37 \pm 0.9 \mathrm{a}$ & $1.55 \pm 0.5 \mathrm{a}$ & $1.01 \pm 0.2 \mathrm{a}$ & $1.32 \pm 0.3 \mathrm{a}$ \\
\hline \multicolumn{7}{|c|}{ Anthocyans $\left(\mathrm{mg} \mathrm{L}^{-1}\right.$ malvidin) } \\
\hline Delphinidin 3-O-glucoside & $4.89 \pm 1.3 \mathrm{ab}$ & $3.57 \pm 1.0 \mathrm{~b}$ & $7.1 \pm 1.9 \mathrm{a}$ & $4.55 \pm 1.3 \mathrm{~b}$ & $4.09 \pm 1.1 \mathrm{~b}$ & $2.53 \pm 0.9 \mathrm{~b}$ \\
\hline Cyanidin 3-O-glucoside & $0.4 \pm 0.1 \mathrm{ab}$ & $0.37 \pm 0.1 \mathrm{ab}$ & $0.52 \pm 0.1 \mathrm{a}$ & $0.42 \pm 0.1 \mathrm{ab}$ & $0.44 \pm 0.1 \mathrm{ab}$ & $0.32 \pm 0.1 \mathrm{~b}$ \\
\hline Peonidin 3-O-glucoside & $5.75 \pm 1.2 \mathrm{ab}$ & $4.85 \pm 1.2 \mathrm{~b}$ & $7.89 \pm 1.8 \mathrm{a}$ & $5.71 \pm 1.3 \mathrm{ab}$ & $4.52 \pm 1.5 \mathrm{~b}$ & $3.46 \pm 0.9 \mathrm{~b}$ \\
\hline Petunidin 3-O-glucoside & $8.82 \pm 1.7 \mathrm{~b}$ & $6.85 \pm 1.7 \mathrm{bc}$ & $12.44 \pm 2.4 \mathrm{a}$ & $8.26 \pm 2.0 \mathrm{~b}$ & $6.41 \pm 1.9 \mathrm{bc}$ & $4.57 \pm 1.1 \mathrm{c}$ \\
\hline Malvidin 3- $O$-glucoside & $70.83 \pm 4.1 \mathrm{~b}$ & $62.92 \pm 3.5 \mathrm{c}$ & $80.88 \pm 3.2 \mathrm{a}$ & $64.97 \pm 4.2 \mathrm{bc}$ & $70.37 \pm 3.9 \mathrm{~b}$ & $40.89 \pm 3.5 \mathrm{~d}$ \\
\hline Total glucosides (Glc) & $90.69 \pm 1.7 \mathrm{~b}$ & $78.6 \pm 1.5 \mathrm{~d}$ & $108.83 \pm 1.9 \mathrm{a}$ & $83.91 \pm 1.8 \mathrm{c}$ & $85.83 \pm 1.7 \mathrm{c}$ & $51.77 \pm 1.3 \mathrm{e}$ \\
\hline Peonidin 3-O-acetylglucoside & $0.29 \pm 0.1 \mathrm{a}$ & $0.26 \pm 0.1 \mathrm{a}$ & $0.28 \pm 0.1 \mathrm{a}$ & $0.26 \pm 0.1 \mathrm{a}$ & $0.27 \pm 0.1 \mathrm{a}$ & $0.27 \pm 0.1 \mathrm{a}$ \\
\hline Malvidin 3-O- acetylglucoside & $2.12 \pm 1.3 \mathrm{ab}$ & $1.92 \pm 0.4 \mathrm{ab}$ & $2.64 \pm 1.0 \mathrm{a}$ & $2.0 \pm 0.9 \mathrm{ab}$ & $1.31 \pm 0.2 \mathrm{bc}$ & $0.44 \pm 0.1 \mathrm{c}$ \\
\hline Total acetylglucosides (AcGlc) & $2.41 \pm 0.7 \mathrm{ab}$ & $2.18 \pm 0.3 \mathrm{ab}$ & $2.92 \pm 0.6 \mathrm{a}$ & $2.26 \pm 0.5 \mathrm{ab}$ & $1.58 \pm 0.2 \mathrm{~b}$ & $0.71 \pm 0.1 \mathrm{c}$ \\
\hline Peonidin 3- $O$-coumaroylglucoside & $1.23 \pm 0.8 \mathrm{ab}$ & $0.99 \pm 0.1 \mathrm{ab}$ & $1.6 \pm 0.2 \mathrm{a}$ & $1.07 \pm 0.2 \mathrm{ab}$ & $1.2 \pm 0.1 \mathrm{ab}$ & $0.72 \pm 0.09 \mathrm{~b}$ \\
\hline Malvidin 3-O-coumaroylglucoside & $5.29 \pm 1.6 \mathrm{ab}$ & $3.99 \pm 1.1 \mathrm{bc}$ & $6.97 \pm 1.5 \mathrm{a}$ & $4.35 \pm 1.0 \mathrm{bc}$ & $2.55 \pm 0.9 \mathrm{c}$ & $2.55 \pm 0.7 \mathrm{c}$ \\
\hline Total coumaroyl-glucosides (coum Glc) & $6.52 \pm 1.2 \mathrm{~b}$ & $4.98 \pm 0.6 \mathrm{~cd}$ & $8.57 \pm 0.8 \mathrm{a}$ & $5.42 \pm 0.6 \mathrm{bc}$ & $3.75 \pm 0.5 \mathrm{de}$ & $3.27 \pm 0.4 \mathrm{e}$ \\
\hline Total anthocyan-monoglycosides & $99.62 \pm 1.2 \mathrm{~b}$ & $85.72 \pm 0.8 \mathrm{~d}$ & $120.30 \pm 1.1 \mathrm{a}$ & $91.59 \pm 0.9 \mathrm{c}$ & $91.16 \pm 0.8 \mathrm{c}$ & $55.75 \pm 0.6 \mathrm{e}$ \\
\hline$\sum$ Glc $/ \sum$ AcGlc & $37.63 \pm 2.4 \mathrm{c}$ & $36.04 \pm 5.0 \mathrm{c}$ & $37.27 \pm 3.1 \mathrm{c}$ & $37.13 \pm 3.6 \mathrm{c}$ & $54.32 \pm 8.5 \mathrm{~b}$ & $72.92 \pm 13.0 \mathrm{a}$ \\
\hline$\Sigma$ Glc/ $/$ coumGlc & $13.91 \pm 1.4 \mathrm{~b}$ & $15.78 \pm 2.5 \mathrm{~b}$ & $12.70 \pm 2.3 \mathrm{~b}$ & $15.48 \pm 3.0 \mathrm{~b}$ & $22.89 \pm 3.4 \mathrm{a}$ & $15.83 \pm 3.2 \mathrm{~b}$ \\
\hline$\sum$ AcGlc $/ \Sigma$ coumGlc & $0.37 \pm 0.1 \mathrm{ab}$ & $0.44 \pm 0.1 \mathrm{a}$ & $0.34 \pm 0.1 \mathrm{ab}$ & $0.42 \pm 0.1 \mathrm{a}$ & $0.42 \pm 0.1 \mathrm{a}$ & $0.22 \pm 0.1 \mathrm{~b}$ \\
\hline
\end{tabular}

Average values \pm standard errors $(n=3)$. The letters in the brackets show the statistical difference among results for $\mathrm{p}<0.05$. For the same compound, a common letter for 2 or more variants shows no significant difference among them. 
Table 2. Phenolic composition of wines obtained in experimental variants of 'Fetească neagră', vintage 2017

\begin{tabular}{|c|c|c|c|c|c|c|}
\hline \multirow[b]{2}{*}{ Compound } & \multicolumn{2}{|c|}{$\mathrm{C}=28$ buds } & \multicolumn{2}{|c|}{$\mathrm{T} 1=20$ buds } & \multicolumn{2}{|c|}{$\mathrm{T} 2=36$ buds } \\
\hline & $\begin{array}{l}\mathrm{V} 1=8 \text { days } \\
\text { maceration }\end{array}$ & $\begin{array}{l}\mathrm{V} 2=16 \text { days } \\
\text { maceration }\end{array}$ & $\begin{array}{l}\mathrm{V} 1=8 \text { days } \\
\text { maceration }\end{array}$ & $\begin{array}{l}\mathrm{V} 2=16 \text { days } \\
\text { maceration }\end{array}$ & $\begin{array}{l}\mathrm{V}=8 \text { days } \\
\text { maceration }\end{array}$ & $\begin{array}{l}\mathrm{V} 2=16 \text { days } \\
\text { maceration }\end{array}$ \\
\hline \multicolumn{7}{|c|}{ General composition } \\
\hline Alcoholic strength (\% vol.) & $11.9 \pm 0.1 \mathrm{~b}$ & $12.1 \pm 0.2 \mathrm{~b}$ & $12.6 \pm 0.1 \mathrm{a}$ & $12.7 \pm 0.2 \mathrm{a}$ & $11.2 \pm 0.2 \mathrm{c}$ & $11.4 \pm 0.1 \mathrm{c}$ \\
\hline Reducing sugar $\left(\mathrm{g} \mathrm{L}^{-1}\right)$ & $2.2 \pm 0.1 \mathrm{~b}$ & $2.2 \pm 0.1 \mathrm{~b}$ & $2.5 \pm 0.1 \mathrm{a}$ & $2.3 \pm 0.2 \mathrm{ab}$ & $2.4 \pm 0.2 \mathrm{ab}$ & $2.2 \pm 0.1 \mathrm{~b}$ \\
\hline Total acidity ( $\mathrm{g} \mathrm{L}^{-1}$ tartaric acid) & $6.26 \pm 0.20 \mathrm{~b}$ & $6.75 \pm 0.24 \mathrm{a}$ & $6.43 \pm 0.20 \mathrm{ab}$ & $6.75 \pm 0.18 \mathrm{a}$ & $6.25 \pm 0.21 \mathrm{~b}$ & $6.83 \pm 0.26 \mathrm{a}$ \\
\hline Volatile acidity (g L ${ }^{-1}$ acetic acid) & $0.41 \pm 0.13 \mathrm{a}$ & $0.42 \pm 0.11 \mathrm{a}$ & $0.44 \pm 0.14 \mathrm{a}$ & $0.45 \pm 0.10 \mathrm{a}$ & $0.43 \pm 0.10 \mathrm{a}$ & $0.45 \pm 0.12 \mathrm{a}$ \\
\hline Total dry extract $\left(\mathrm{g} \mathrm{L}^{-1}\right)$ & $26.4 \pm 0.3 \mathrm{c}$ & $27.0 \pm 0.2 \mathrm{~b}$ & $27.8 \pm 0.2 \mathrm{a}$ & $27.8 \pm 0.1 \mathrm{a}$ & $25.2 \pm 0.3 \mathrm{~d}$ & $25.2 \pm 0.1 \mathrm{~d}$ \\
\hline Non-reducing dry extract $\left(\mathrm{g} \mathrm{L}^{-1}\right)$ & $24.2 \pm 0.2 \mathrm{c}$ & $24.8 \pm 0.1 \mathrm{~b}$ & $25.3 \pm 0.1 \mathrm{a}$ & $25.5 \pm 0.2 \mathrm{a}$ & $22.8 \pm 0.2 \mathrm{~d}$ & $23.0 \pm 0.2 \mathrm{~d}$ \\
\hline Total $\mathrm{SO}_{2}\left(\mathrm{mg} \mathrm{L}^{-1}\right)$ & $72.5 \pm 3.8 \mathrm{~b}$ & $78.4 \pm 3.0 \mathrm{a}$ & $70.5 \pm 2.4 \mathrm{~b}$ & $74.5 \pm 2.8 \mathrm{a}$ & $78.2 \pm 2.2 \mathrm{a}$ & $79.1 \pm 2.7 \mathrm{a}$ \\
\hline Free $\mathrm{SO}_{2}\left(\mathrm{mg} \mathrm{L}^{-1}\right)$ & $22.8 \pm 2.4 \mathrm{ab}$ & $23.4 \pm 2.1 \mathrm{ab}$ & $22.5 \pm 2.0 \mathrm{~b}$ & $24.8 \pm 2.4 \mathrm{ab}$ & $25.2 \pm 2.5 \mathrm{ab}$ & $27.2 \pm 2.2 \mathrm{a}$ \\
\hline \multicolumn{7}{|c|}{ Phenolic compounds } \\
\hline \multicolumn{7}{|c|}{ Phenolic acids (mg L $\left.{ }^{-1}\right)$} \\
\hline Gallic acid & $33.08 \pm 2.9 \mathrm{~d}$ & $49.81 \pm 4.1 \mathrm{~b}$ & $36.34 \pm 3.2 \mathrm{~d}$ & $57.44 \pm 4.2 \mathrm{a}$ & $30.86 \pm 2.9 \mathrm{~d}$ & $43.34 \pm 3.2 \mathrm{c}$ \\
\hline p-Hydroxybenzoic acid & $0.4 \pm 0.1 \mathrm{bc}$ & $0.66 \pm 0.2 \mathrm{~b}$ & $0.62 \pm 0.2 \mathrm{~b}$ & $0.97 \pm 0.1 \mathrm{a}$ & $0.31 \pm 0.1 \mathrm{c}$ & $0.45 \pm 0.1 \mathrm{bc}$ \\
\hline p-Coumaric acid & $1.2 \pm 0.3 \mathrm{ab}$ & $0.15 \pm 0.02 c$ & $1.56 \pm 0.8 \mathrm{a}$ & $1.15 \pm 0.4 \mathrm{ab}$ & $1.01 \pm 0.02 \mathrm{ab}$ & $0.69 \pm 0.1 \mathrm{bc}$ \\
\hline Ferulic acid & $0.03 \pm 0.01 \mathrm{~d}$ & $0.09 \pm 0.01 \mathrm{ab}$ & $0.07 \pm 0.01 \mathrm{bc}$ & $0.10 \pm 0.02 \mathrm{a}$ & $0.03 \pm 0.01 \mathrm{~d}$ & $0.06 \pm 0.02 \mathrm{c}$ \\
\hline \multicolumn{7}{|c|}{ Flavanols $\left(m g L^{-1}\right)$} \\
\hline Catechin & $4.83 \pm 1.5 \mathrm{~cd}$ & $7.84 \pm 1.7 \mathrm{ab}$ & $5.75 \pm 1.4 \mathrm{abc}$ & $8.07 \pm 1.8 \mathrm{a}$ & $2.58 \pm 0.5 \mathrm{~d}$ & $5.23 \pm 1.2 \mathrm{bc}$ \\
\hline Epicatechin & $4.24 \pm 1.2 \mathrm{~b}$ & $6.44 \pm 1.1 \mathrm{ab}$ & $4.91 \pm 1.2 \mathrm{~b}$ & $7.83 \pm 1.5$ & $4.03 \pm 1.0 \mathrm{~b}$ & $5.88 \pm 1.5 \mathrm{ab}$ \\
\hline \multicolumn{7}{|c|}{ Flavonols $\left(m g L^{-I}\right)$} \\
\hline Myricetin & $2.06 \pm 0.9 \mathrm{ab}$ & $0.37 \pm 0.1 \mathrm{c}$ & $3.0 \pm 1.0 \mathrm{a}$ & $1.23 \pm 0.5 \mathrm{bc}$ & $1.86 \pm 0.6 \mathrm{ab}$ & $0.93 \pm 0.2 \mathrm{bc}$ \\
\hline Quercetin & $0.2 \pm 0.05 \mathrm{c}$ & $0.15 \pm 0.09 \mathrm{c}$ & $1.15 \pm 0.2 \mathrm{a}$ & $0.39 \pm 0.1 \mathrm{~b}$ & $0.10 \pm 0.02 \mathrm{c}$ & $0.03 \pm 0.01 \mathrm{c}$ \\
\hline \multicolumn{7}{|c|}{ Stilbens $\left(m g L^{-I}\right)$} \\
\hline Trans-resveratrol & $3.64 \pm 1.1 \mathrm{c}$ & $5.48 \pm 1,6 \mathrm{bc}$ & $6.23 \pm 1,3 \mathrm{~b}$ & $9.29 \pm 2,1 \mathrm{a}$ & $2.99 \pm 0,2 \mathrm{c}$ & $4.96 \pm 0,8 \mathrm{bc}$ \\
\hline \multicolumn{7}{|c|}{ Anthocyans (mg $L^{-1}$ malvidina) } \\
\hline Delphinidin 3-O-glucoside & $0.85 \pm 0.1 \mathrm{~b}$ & $0.35 \pm 0.02 \mathrm{~b}$ & $2.43 \pm 0.8 \mathrm{a}$ & $1.93 \pm 0.3 \mathrm{a}$ & $0.38 \pm 0.1 \mathrm{~b}$ & $0.22 \pm 0.09 \mathrm{~b}$ \\
\hline Cyanidin 3-O-glucoside & $0.31 \pm 0.09 \mathrm{~b}$ & $0.28 \pm 0.1 \mathrm{~b}$ & $1.06 \pm 0.2 \mathrm{a}$ & $1.08 \pm 0.3 \mathrm{a}$ & $0.24 \pm 0.1 \mathrm{~b}$ & $0.86 \pm 0.1 \mathrm{a}$ \\
\hline Peonidin 3-O-glucoside & $0.61 \pm 0.1 \mathrm{~b}$ & $0.37 \pm 0.09 \mathrm{~b}$ & $2.27 \pm 0.3 \mathrm{a}$ & $2.38 \pm 0.8 \mathrm{a}$ & $0.25 \pm 0.1 \mathrm{~b}$ & $0.12 \pm 0.02 \mathrm{~b}$ \\
\hline Petunidin 3-O-glucoside & $2.28 \pm 1.0 \mathrm{ab}$ & $0.74 \pm 0.1 \mathrm{c}$ & $3.17 \pm 1.1 \mathrm{a}$ & $2.09 \pm 0.3 \mathrm{ab}$ & $1.9 \pm 0.2 \mathrm{bc}$ & $1.43 \pm 0.1 \mathrm{bc}$ \\
\hline Malvidin 3- $O$-glucoside & $32.12 \pm 3.2 \mathrm{c}$ & $20.72 \pm 2.3 \mathrm{~d}$ & $49.03 \pm 2.5 \mathrm{a}$ & $41.79 \pm 2.9 \mathrm{~b}$ & $23.4 \pm 1.1 \mathrm{~d}$ & $16.36 \pm 1.1 \mathrm{e}$ \\
\hline Total glucosides (Glc) & $36.17 \pm 0.7 \mathrm{c}$ & $22.46 \pm 0.5 \mathrm{e}$ & $57.96 \pm 0.9 \mathrm{a}$ & $49.27 \pm 0.9 \mathrm{~b}$ & $26.17 \pm 0.1 \mathrm{~d}$ & $18.99 \pm 0.3 \mathrm{f}$ \\
\hline Peonidin 3-O-acetylglucoside & $0.31 \pm 0.1 \mathrm{a}$ & $0.26 \pm 0.1 \mathrm{a}$ & $0.43 \pm 0.1 \mathrm{a}$ & $0.42 \pm 0.1 \mathrm{a}$ & $0.42 \pm 0.1 \mathrm{a}$ & $0.4 \pm 0.1 \mathrm{a}$ \\
\hline Malvidin 3-O- acetylglucoside & $1.11 \pm 0.3 \mathrm{bc}$ & $0.55 \pm 0.1 \mathrm{c}$ & $2.7 \pm 1.1 \mathrm{a}$ & $2.02 \pm 0.9 \mathrm{ab}$ & $1.46 \pm 0.2 \mathrm{bc}$ & $1.4 \pm 0.3 \mathrm{bc}$ \\
\hline Total acetyl-glucosides (AcGlc) & $1.42 \pm 0.2 \mathrm{c}$ & $0.81 \pm 0.1 \mathrm{~d}$ & $3.13 \pm 0.6 \mathrm{a}$ & $2.44 \pm 0.5 \mathrm{~b}$ & $1.88 \pm 0.2 \mathrm{bc}$ & $1.8 \pm 0.2 \mathrm{bc}$ \\
\hline Peonidin 3- $O$-coumaroylglucoside & $0.25 \pm 0.08 \mathrm{~b}$ & $0.2 \pm 0.07 \mathrm{~b}$ & $0.88 \pm 0.2 \mathrm{a}$ & $0.76 \pm 0.1 \mathrm{a}$ & $0.19 \pm 0.08 \mathrm{~b}$ & $0.16 \pm 0.02 \mathrm{~b}$ \\
\hline Malvidin 3-O-coumaroylglucoside & $1.29 \pm 0.4 \mathrm{~b}$ & $0.49 \pm 0.09 \mathrm{~b}$ & $2.68 \pm 0.8 \mathrm{a}$ & $2.56 \pm 0.9 \mathrm{a}$ & $1.19 \pm 0.1 \mathrm{~b}$ & $1.11 \pm 0.1 \mathrm{~b}$ \\
\hline Total coumaroy-Iglucosides (coum Glc) & $1.54 \pm 0.2 \mathrm{~b}$ & $0.69 \pm 0.08 \mathrm{c}$ & $3.56 \pm 0.5 \mathrm{a}$ & $3.32 \pm 0.5 \mathrm{a}$ & $1.38 \pm 0.09 \mathrm{~b}$ & $1.27 \pm 0.06 \mathrm{~b}$ \\
\hline Total anthocyan-monoglycosides & $39.13 \pm 0.4 \mathrm{c}$ & $23.69 \pm 0.2 \mathrm{e}$ & $64.65 \pm 0.6 \mathrm{a}$ & $55.03 \pm 0.6 \mathrm{~b}$ & $29.43 \pm 0.1 \mathrm{~d}$ & $22.06 \pm 0.2 \mathrm{f}$ \\
\hline$\sum$ Glc $/ \sum$ AcGlc & $25.47 \pm 1.5 \mathrm{a}$ & $27.73 \pm 2.0 \mathrm{a}$ & $18.52 \pm 1.3 \mathrm{~b}$ & $20.19 \pm 1.6 \mathrm{~b}$ & $13.92 \pm 1.0 \mathrm{c}$ & $10.55 \pm 1.1 \mathrm{~d}$ \\
\hline$\Sigma$ Glc/ $/$ coumGlc & $23.49 \pm 1.8 \mathrm{~b}$ & $32.55 \pm 2.1 \mathrm{a}$ & $16.28 \pm 1.0 \mathrm{~cd}$ & $14.84 \pm 1.2 \mathrm{~d}$ & $18.96 \pm 1.5 \mathrm{c}$ & $14.95 \pm 1.5 \mathrm{~d}$ \\
\hline$\sum$ AcGlc $/ \Sigma$ coumGlc & $0.92 \pm 0.1 \mathrm{c}$ & $1.17 \pm 0.1 \mathrm{~b}$ & $0.88 \pm 0.1 \mathrm{~cd}$ & $0.73 \pm 0.1 \mathrm{~d}$ & $1.36 \pm 0.1 \mathrm{a}$ & $1.42 \pm 0.1 \mathrm{a}$ \\
\hline
\end{tabular}

Average values \pm standard errors $(n=3)$. The letters in the brackets show the statistical difference among results for $\mathrm{p}<0.05$. For the same compound, a common letter for 2 or more variants shows no significant difference among them.

The other parameters, volatile acidity and the concentrations of free and total sulphur dioxide, are mostly related to the winemaking technology and although very correlated with the final wine quality, they are not supposed to be influenced by the grape-yield. Thus, the values determined are rightfully not significantly different among these variants. The low values of volatile acidity, $0.41-0.49 \mathrm{~g} \mathrm{~L}^{-1}$ acetic acid, demonstrate that the alcoholic and malolactic fermentations were very well controlled, even in the presence of low quantities of total sulphur dioxide, namely around $75 \mathrm{mg} \mathrm{L}^{-1}$. These are very good values for organic wines, in which, by law should not exceed $130 \mathrm{mg} \mathrm{L}^{-1}$ for red wines with more than $2 \mathrm{~g} \mathrm{~L}^{-1}$ sugar (Reg. EU 203, 2012).

The polyphenolic profile of 'Fetească neagră', obtained by HPLC, shows the presence in wines of important levels of phenolic acids, flavonols, flavanones, stilbens and anthocyanins, which are presented in detail in Tables 1 and 2. To assess the influence of the independent factors on the polyphenol accumulation and distribution, a PCA analysis was performed and the results are presented in Figure 1a-d. 

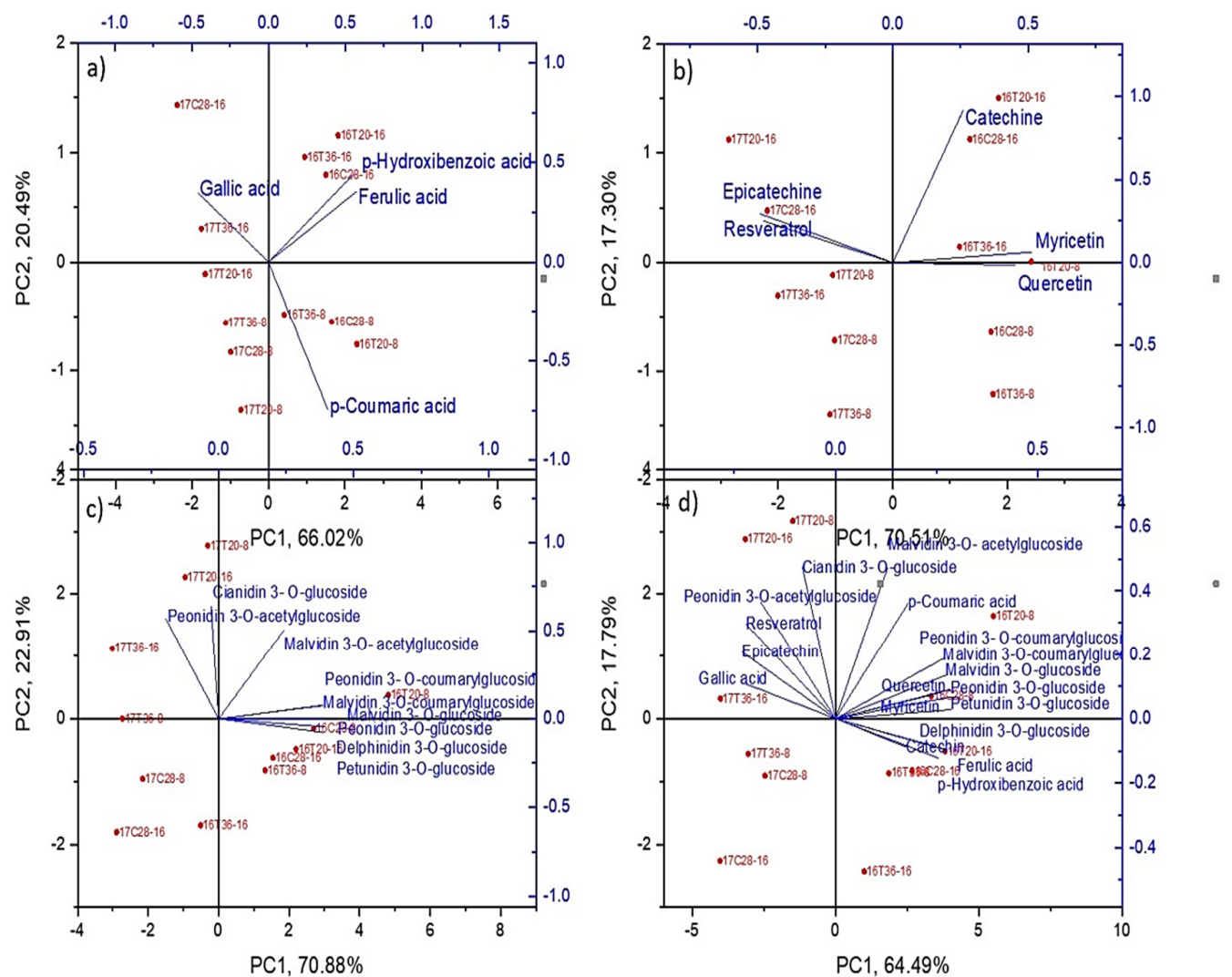

Figure 1. Principal Component Analysis (PCA) showing the components which explain the variation of the samples obtained in 2016 and 2017: (a) Phenolic acids; (b) Flavonols, flavanols and stilbenes; (c) Anthocyanin derivatives; (d) All polyphenols

Distribution of the polyphenols depending on the vintage (prefix 16 or 17 for 2016 or 2017), bud-load/vine (T20, C28, T36 for loads of 20,28 and 36 bud/vine) or maceration time (suffix- 8 or 16 for the days of maceration)

From the phenolic acids class, as reported by other authors too, both on 'Fetească neagră' (Geana et al., 2011) and other varieties (Porgall et al., 2012), gallic acid predominates, with values ranging in our experiment from 9.77 to $57.44 \mathrm{mg} \mathrm{L}^{-1}$, higher values being present in 2017 (Figure 1a). Its concentration increases with the time of maceration during winemaking (Figure 1a), while the grape yield has less influence on this parameter. The other phenolic acids, p-hydroxybenzoic, p-coumaric and ferulic, tend to have an inverse accumulation with the gallic acid (Figure 1a), their values being higher in 2016, a more favourable year for grape quality.

As regards the flavanols, their values also increased with the maceration time, especially in the case of catechin, for which the concentration almost doubled in eight days of additional maceration. This is in agreement with other authors' observations who reported increase in catechins and proanthocyanidins in 14 days of maceration on skins in red wines (Kovac et al., 1992). As shown in Figure1b, the catechin level was higher in favourable years (2016), while epicatechin increased in less favourable years (2017). From the flavonol class myricetin and quercetin higher concentrations are also correlated with the favourable 2016 vintage, while the stilbene and trans-resveratrol tended to accumulate more in the less favourable 2017 (Figure 1b). The extraction was significantly increased by the longer maceration on skins (Figure $1 \mathrm{~b}$ ) whenever the specific phenol was present in sufficient quantities on the skins. As we can see for the case of resveratrol, in 2016 its levels were relatively low, around $1 \mathrm{mg} \mathrm{L}^{-1}$ (Table 1), thus its increase toward $1.5 \mathrm{mg} \mathrm{L}^{-1}$ through longer maceration did not attain significance level, while in 2017, the resveratrol increased from $2.99-6.23 \mathrm{mg} \mathrm{L}^{-1}$ to 4.96-9.29 $\mathrm{mg} \mathrm{L}^{-1}$ in eight supplementary days of maceration (Table 2). In accordance to other authors too, 
when present in skins, the resveratrol extraction can be increased even by 13 times by maceration as compared to the values in the free-run musts (Jeandet et al., 1995).

The anthocyanins, the red pigments of wine, influence the colour shade and intensity through their concentration and their proportion, each anthocyanin having colours which depend on their chemical structure and external factors, such as the $\mathrm{pH}$ or sulphur dioxide. Thus, any change in the anthocyanin profile leads to colour change. Obviously, in the more favourable year, 2016, the anthocyanin accumulation was much higher, especially as regards the malvidin 3-O-glucoside, which is the most representative anthocyanin found in 'Fetească neagră'. Malvidin 3-O-glucoside content is approximately double in 2016, with an average value of $65.14 \mathrm{mg} \mathrm{L}^{-1}$ as compared to 2017 , when the average was $30.57 \mathrm{mg} \mathrm{L}^{-1}$. This happened with all the other determined anthocyanins, with the exception of cyanidin-3-O-glucoside and peonidin 3-O-acetylglucoside (Figure 1c), which had a slight increase in 2017 as compared to 2016, but their values are too small to influence the overall colour. The other anthocyanins accumulated better in warmer years, so that the initial values of delphinidin, peonidin, petunidin were several times higher in the favourable vintage of 2016 (Table 1), than in 2017 (Table 2). Anthocyanins is the class of polyphenols which is the most influenced by the grape yield, the variant with the reduced yield, $20 \mathrm{bud} /$ vine, having the higher anthocyanin concentration (Figure 1c). The longer maceration period, 16 days, decreased the content of malvidin derivatives by an average of $25.5 \%$. The other free anthocyanins decreased as well during maceration (Tables 1 and 2), due to polymerisation or condensation with other polyphenols. This fact was confirmed by other previous studies on other grape varieties (Herjavec et al., 2012).

Overall, it can be observed from the PCA analyses (Figure $1 \mathrm{a}-\mathrm{d}$ ), that the less favourable year (2017) was characterized by an increase in gallic acid, epicatechin, resveratrol and a decrease in catechine, myricetin and quercetin, ferulic, p-hydroxibenzoic and p-coumaric acids and, especially, in most of the colour compounds. As displayed in Figure 1d the samples of 2017 and 2016 are differentiated mostly based on the red-coloured phenolic compounds, which are included in the Principal Component 1, which accounts for 64.69\% of the total variance. Most other determined phenolic compounds, from the classes of phenolic acids, flavanols, flavonols and stilbenes are included in the Principal Component 2, which explains only $17.79 \%$ of the variance.

\section{Conclusions}

This study showed that the quality of the organic wines depends highly on the climatic conditions and that the most influence on the chemical parameters was exerted by the year of harvest. However, the yield reduction, coupled with prolongation of the maceration time on skins during winemaking, especially in the less favourable years, can lead to better accumulation of sugars, of the colour pigments and other polyphenols (ferulic acid, p-coumaric acid and p-hydroxybenzoic acid, catechin, myricetin, quercetin). The accumulation of trans-resveratrol, a stilbene correlated with many health benefits in humans, is lower in favourable climatic years (average of $1.1 \mathrm{mg} \mathrm{L}^{-1}$ ), therefore, even though maceration increases its extraction (average of $1.45 \mathrm{mg} \mathrm{L}^{-1}$ ), the increase is insignificant. However, in less favourable years resveratrol accumulates in higher quantities (average $4.28 \mathrm{mg} \mathrm{L}^{-1}$ ) and its concentration in wine can be increased by longer maceration on skins, up to an average of 6.57 $\mathrm{mg} \mathrm{L}^{-1}$. Thus, especially in the less favourable years, interventions in the vineyard and in winemaking process are necessary to optimize the content of quality-related compounds. 


\section{Authors' Contributions}

Conceptualization: VA; Data curation: VA; Formal analysis: EIG; Investigation: AOA; Methodology: EIG; Software: AR; Supervision: AOA; Validation: AOA; Writing - original draft: VA, AOA; Writing - review and editing: AOA. All authors read and approved the final manuscript.

\section{Acknowledgements}

This work was supported by the Ministry of Agriculture and Rural Development Bucharest, Sectorial Plan under ADER 3.3.10 Project.

\section{Conflict of Interests}

The authors declare that there are no conflicts of interest related to this article.

\section{References}

Antoce AO (2007). Oenology, chemistry and sensory analysis. Universitaria Printing House, Craiova, Romania.

Antoce AO, Cojocaru G (2018). Characterization of quality potential of Feteasca neagra grapes cultivated in different Romanian wine region. Proceeding of the Conference Agriculture for Life, Life for Agriculture, pp 238-243.

Antoce AO (2019). A SWOT evaluation of organic winemaking regulatory implications in the support for organic products in Romania. Scientific Papers. Series B, Horticulture 63:235-246. http://horticulturejournal.usamv.ro/index.php/scientific-papers/issues?id=668

Antoce AO, Stockley C (2019). An overview of the implications of wine on human health, with special consideration of the wine-derived phenolic compounds. AgroLife Scientific Journal 8:21-34. http://agrolifejournal.usamv.ro

ASRO (2012). Romanian Association for Standardization, http://www.asro.ro/.

Busuricu F, Negreanu-Parjol T, Popescu A, Margaritti D, Lupu C, Schroder V, Tomas S (2013). Evaluation of the antioxidant activity of certain Romanian wines. Journal of Environmental Protection and Ecology 14:18281835. https://www.cabdirect.org/cabdirect/abstract/20153052146

Colibaba LC, Scuturasu C, Codreanu M, Calin I, Luchian CE, Cotea VV (2019). Data on changes in wine phenolic compounds, biogenic amines and amino acids composition after treatment with Carbon-based Materials. Proceeding of the $7^{\text {th }}$ International Conference E-Health and Bioengineering, pp 1-4

Dobrinas S, Soceanu A, Artem V (2012). Determination of polyphenolic compounds in Murfatlar wines by validated spectrophotometric method. Environmental Engineering and Management Journal 14:863-870. http://dx.doi.org/10.30638/eemj.2015.097

Genc M, Genc S, Goksungur Y (2017). Exergy analysis of wine production: Red wine production process as a case study. Applied Thermal Engineering 117:511-521. https://doi.org/10.1016/j.applthermaleng.2017.02.009

Geana IE, Popescu R, Costinel D, Dinca OR, Stefanescu I, Ionete RE, Bala C (2016). Verifying the red wines adulteration through isotopic and chromatografic investigation coupled with multivariate statistic interpretation of the data. Food Control 62:1-9. https://doi.org/10.1016/j.foodcont.2015.10.003

Geana IE, Ionete RE, Tudorache A, Pasa R, Postolache E, Ranca A (2011). Phenolic contents of Romanian wines with different geographical origins. Asian Journal of Chemistry 23:10718. http://dx.doi.org/10.15835/buasvmcnhort:4951

Ignat G, Colibaba C, Costuleanu C L, Timofte AA, Sandu I, Moraru I, Ungureanu G, Bejenaru C (2017). Management of maceration-fermentation technologies regarding the antioxidant profiles of some wines from Iasi vineyard. Revista de Chimie 68:2922-2924. https://doi.org/10.37358/RC.17.12.6007

Hussain T, Tan B, Yin Y, Blachier F, Tossou MCB, Rahu N (2016). Oxidative stress and inflammation: what polyphenols can do for us? Oxidative Medicine Cellular Longevity 2016:7432797. https://doi.org/10.1155/2016/7432797 
Herjavec S, Jeromel A, Maslov L, Jagatic Korenika AM, Mihaljevic M, Prusina T (2012). Influence of different maceration times on the anthocyanin composition and sensory properties of Blatina wines. Agriculturae Conspectus Scientificus 77:41-44. https://hrcak.srce.hr/file/115720

Jeandet P, Bessis R, Maume BF, Meunier P, Peyron D, Trollat P (1995). Effect of enological practices on the resveratrol isomer content of wine. Journal of Agricultural and Food Chemistry 43:316-319. https://doi.org/10.1021/jf00050a010

Kovac V, Alonso E, Bourzeix M, Revilla E (1992). Effect of several enological practices on the content of catechins and proanthocyanidins in red wines. Journal of Agricultural and Food Chemistry 40:1953-1957. https://doi.org/10.1021/jf00022a045

Luchian CE, Colibaba CL, Codreanu M, Tudose-Sandu-Ville Ş, Niculaua M, Cotea VV (2018). Assay of antioxidant capacity and phenolic compounds in some Romanian and Cypriot wine. Notulae Botanicae Horti Agrobotanici Cluj-Napoca, 46(1):240-246. https://doi.org/10.15835/nbha46110885

OIV (2008). Determination of nine major anthocyanins in red and rosé wines using HPLC. Compendium of international methods of analysis of wine and musts. Vol. 2, International Organisation of Vine and Wine, pp 48-60. Retrieved 2021 February 15 from https://www.oiv.int/public/medias/7787/oiv-compendium-ofinternational-methods-of-analysis-vol1-en.pdf

Ozabagaoglu MO, Akyol A, Ozay A (2007). The demand for organic wines and organic wine marketing. Journal of Envirnmental Protection and Ecology 8:171-178. https://www.researchgate.net

Petrescu DC, Petrescu-Mag RM, Burny P (2015). Management of environmental security through organic agriculture. Contribution of consumer behaviour. Engineering and Management Journal 14:2625-2636. http://hdl.handle.net/2268/192148

Porgali E, Buyuktuncel E (2012). Determination of phenolic composition and antioxidant capacity of native red wines by high performance liquid chromatography and spectrophotometric methods. Food Research International 45:145-154. http://dx.doi.org/10.1016/j.foodres.2011.10.025

Rahmani M, Bakhshi D, Qolov M (2012). Impact of pruning severity and training systems on red and white seedless table grape (Vitis vinifera) qualitative indices. Australian Journal of Crop Science 9:55-61. https://www.researchgate.net

Willer H, Lernoud J (2017). Organic Viticulture Worldwide 2015. Retrieved 2021 February 16 from https://orgprints.org/id/eprint/31416/1/willer-lernoud-2017-viticulture-2015.pdf

Reg. (EU) (2012). Commission Implementing Regulation (EU) No 203 of 8 March 2012 amending Reg. (EC) No 889/2008 laying down detailed rules for the implementation of Council Regulation (EC) No 834/2007, as regards detailed rules on organic wine. Retrieved 2021 February 18 from https://eur-lex.europa.eu/legalcontent/EN/TXT/PDF/?uri=CELEX:32012R0203\&from $=E N$

Reg. (EU) (2018). Regulation (EU) 848 of the European Parliament and of the Council of 30 May 2018 on organic production and labelling of organic products and repealing Council Regulation (EC) No 834/2007. Retrieved 2021 February 18 from https://eur-lex.europa.eu/legalcontent/EN/TXT/PDF/?uri=CELEX:32018R0848\&from $=E N$

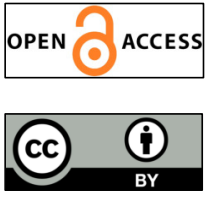

The journal offers free, immediate, and unrestricted access to peer-reviewed research and scholarly work. Users are allowed to read, download, copy, distribute, print, search, or link to the full texts of the articles, or use them for any other lawful purpose, without asking prior permission from the publisher or the author.

License - Articles published in Notulae Botanicae Horti Agrobotanici Cluj-Napoca are Open-Access, distributed under the terms and conditions of the Creative Commons Attribution (CC BY 4.0) License. (c) Articles by the authors; UASVM, Cluj-Napoca, Romania. The journal allows the author(s) to hold the copyright/to retain publishing rights without restriction. 